\title{
Optimization of Sports Good Recycling Management System Based on Internet of Things
}

\author{
Panhong Ren, ${ }^{1}$ Mengjian Nie, ${ }^{2}$ and Hui Ming $\mathbb{D}^{3}$ \\ ${ }^{1}$ Sanquan College of Xinxiang Medical University, Xinxiang 453003, China \\ ${ }^{2}$ Henan Normal University, Xinxiang 453000, China \\ ${ }^{3}$ Xinxiang Medical University, Xinxiang 453003, China \\ Correspondence should be addressed to Hui Ming; 091049@xxmu.edu.cn
}

Received 10 April 2021; Revised 18 May 2021; Accepted 20 May 2021; Published 7 June 2021

Academic Editor: Wei Wang

Copyright (C) 2021 Panhong Ren et al. This is an open access article distributed under the Creative Commons Attribution License, which permits unrestricted use, distribution, and reproduction in any medium, provided the original work is properly cited.

Based on the technology of Internet of Things, this paper proposes a sports good recycling management system and realizes the system optimization. Based on the management of returned good flow based on the Internet of Things, we can comprehensively apply system integration technology, system architecture, cloud computing technology, etc., based on the current situation of surrounding return flow management, combined with the reality of the sports product recycling business process. From two aspects of process information and operation information, based on the information system of each link of the Internet of Things recycling, a recycling logistics information management system platform is constructed. This can solve the problem of the integrated management of the recycling logistics process and the operation control of the recycling logistics management system. In the stage of sports information management, combined with the Internet of Things technology, we should do a good job of sports artificial intelligence management and information knowledge exploration. In the development of sports under the framework of the Internet of Things, we should combine the needs of sports information management and the technology mode of the Internet of Things, so as to realize the scientific and standardized management of sports good recycling under the Internet of Things technology.

\section{Introduction}

As a green industry, the sports industry is called the sunrise industry and the civilian production industry. It is an important part of the modern industrial system. It has a large market demand and integrates related characteristics. It has become a national strategy in the context of national fitness. The development of the sports industry has also reached a new level, becoming the dominant source of power for the promotion of social and economic value. However, it must be admitted that sports goods involve complex links such as production, processing, circulation and sales, and lack of information correlation and sharing before each link. When quality and safety problems are found, products cannot be tracked and traced effectively to find the key to the problem [1]. Therefore, the sports good supply chain should grasp the great opportunity of developing information economy and promoting intelligent application and accelerate the realization of informatization and visual supervision of each link of the supply chain with the support of the new generation of information technology such as the Internet of Things [2]. With the continuous development of technology, robots and the development of the Internet of Things industry has been applied to all walks of life, in sport management industry, however, did not involve, traditional sports goods still stay in the human management stage, although this way of management directly, but brought great inconvenience to people, the most important thing is given at the end of the sports people to drive a little way to return the sports goods, this caused people tired body and mind pressure, then the traditional sporting good length because management is not caused by the loss of sporting goods, such not only increased the economic operating costs. And this increased the difficulty of work for people, hence the need for a comprehensive and fast sports good recycling management system. 
The application of Internet of Things (IoT) technology is mainly to realize effective connection of objects on the basis of agreement, information exchange and communication, and, finally, to realize an intelligent identification process by combining information, information, communication, and sensing. Generally speaking, IoT technology should be mainly used in three layers, that is, the application of the perception layer and the network layer, as well as the application of the processing layer [3]. Based on the Internet of Things technology under a sports information management process, mainly combining with the form of content couplet net perception technology, the sports information acquisition effect rate increases, combined with the high-speed network of a cloud computing platform, a mode of realizing interconnection application, completes the sports information of intelligent control, and makes the realization of real-time monitoring and automated analysis. By combining the form of information collection, we can generate the record and do a good job of the relevant management of the event information, so as to show the effective application of the awareness layer and the network layer in the application of the multisingle bit cooperative supervision. A hardware perception process in the perceptual layer should combine the integration and application of information resource effectiveness, realize a comprehensive analysis of its own activities, combine a body system structure of historical records, and show the effectiveness of data sharing of interconnected body information [4]. The realization of a network layer structure, combined with the function of data backup and data mining, realizes the effective management of sports information. The application process of mass distribution of the Internet of Things shows the root analysis of the intelligent management layer, the effective application of the reweighting technology system, and the integration of the basic situation of the environment, so as to realize the whole exploration process. In a word, sports information management based on the Internet of Things technology, combined with the process of information system design and implementation evaluation, can realize the effective operation and guarantee of the information system. Pay attention to the realization of security management, realize the effective application of organizational information resources, and do a good job in the overall investment and evaluation of information technology. In the process of information system management, combined with the strategic development of organization competition, the management of sports information industry will be promoted comprehensively, and an effective application and management of sports information service industry will be realized.

Based on the above background, this paper firstly analyzes the operation process, network structure, and business links of the recycling logistics. Combined with the characteristics of the Internet of Things technology, it proposes the feasibility of using the Internet of Things technology to optimize the operation process and the management system. Secondly, the actual requirements of information construction of recycling logistics management are analyzed from the perspective of objects involved in recycling logistics, business process, and Internet of Things technology, and the framework of information platform of recycling logistics is designed based on business function requirements. We combined the actual sports to lead the sporting good recycling business. According to the framework developed by the information platform, the platform has achieved good results in the actual recycling business test. This paper proposes a sports good recycling management system based on the Internet of Things technology, which realizes the process informatization and operation informatization by combining the actual sports good recycling business process, integrated application system integration technology, system architecture, and cloud computing technology, etc. This can solve the problem of comprehensive management of the recycling logistics process and operation control of recycling logistics management system, to achieve the scientific and standardized management of sporting good recycling under the Internet of Things technology.

The rest of this paper is organized as follows: the related work is discussed in Section 2. Section 3 elaborates the recycling management mode of sporting good. In Section 4, based on the Internet of Things technology, a sports goods recycling management platform is established. The fifth part carries on the system optimization and the test. Section 6 summarizes the text.

\section{Related Work}

The close combination of Internet of Things technology and modern logistics technology also significantly improves the efficiency of the sports good logistics management and operation process, which is more convenient and safe, thus attracting the interest of researchers. Hou et al. [5] analyzed the influencing factors of sports product recycling management from the perspective of the value of recycled goods, constructed a mathematical model based on the influencing factors, and provided a reference analysis method for the value of different recycled products by using the analytic hierarchy process. Mahmood and Zubairi [6] in combination with the practical recycling logistics problems of lead-acid sporting goods, considering a fully closed recycling system of each production link, such as purchasing, production, scrap, recycling, and reprocessing, built an integrated management for solving minimum cost and emission pollution management cost and a minimum pollution impact model of a multiobjective fuzzy algorithm solution. Ren et al. [7] developed a returned logistics network model which is constructed to simulate the sports good recycling business in India, through a different stimulus allocation mechanism, with comprehensive consideration of forward and reverse returned logistics operation and design recovery system efficiency factors, through the simulation to predict recovery cost, transportation time, and the relationship between the logistics cost and resource use efficiency. Saha et al. [8] developed a returned logistics network of recycling product flow to determine the premise, through the comprehensive positive outlets and recycling outlets, assuming the outlets to recovery and recovery center, and dealing with the finish $\mathrm{H}$ different role infrastructure multilayer two-way integrated logistics network. Angani et al. [9] established the optimization design model of a recycling logistics network based on the 
interaction mode of new and old exchange through the tradein strategy, constructed the 0-1 mixed integer programming model, analyzed the number and location of the set network, and proposed the feasibility analysis of reducing the difficulty of collection by establishing the trade-in method. Zhou et al. [10] comprehensively considered the uncertain elements that caused the recycling demand of sports goods, established a multiperiod and multiobjective model, and solved the problem of logistics operation allocation among recycling outlets. Ingemarsdotter et al. [11] proposed to design a sports good traceability system based on the Internet of Things by starting with the Internet of Things technology, electronic product codes, and other related technologies and first established the corresponding sports good traceability model. Ramly et al. [12] analyzed the overall requirements of the system in depth. Based on the Internet of Things technology, they design each submodule from the aspects of product sales, product quality inspection, and market supervision to ensure that the quality of sporting goods in circulation can meet the market demand.

With the continuous development of technology, the development of the Internet of Things industry has been applied to the sports industry, but in the sports good management, it has not been involved. The traditional sports good management is still in the stage of manual management. Although this management mode is direct, it brings great inconvenience to people. The loss of traditional sporting goods due to poor management not only increases the cost of economic operation, but also increases the difficulty of work for people. Therefore, a comprehensive and fast sporting good management system is needed.

\section{Recycling Management Mode of Sporting Goods}

3.1. Operation Process. The operation process of recycling logistics mainly refers to a series of logistics activities such as the centralized collection of sporting goods, transportation, distribution, classification, processing, and packaging with the help of logistics nodes and networks, discarding users to identify product transportation methods. Related processing enterprises, after effective processing, extract useful resources, materials, or parts and return to the manufacturer for processing; the specific operation process is shown in Figure 1.

Network nodes participating in the sports product recycling logistics operation can be divided into several different nodes according to their functions: recycling network, recycling center, and processing end point. The recycling network is responsible for completing the business links of sports products entering the recycling system. In the process of recycling, it must act as the main node carrying the functions of concentration, classification, and transportation; carry out logistics operations such as classification, storage, and transportation of sports products according to different flow directions; and finally transport them to the processing center. Sports products are used as raw materials at the end of processing, and renewable resources are obtained through processing.
(1) Recycling point: there are two main construction modes of recovery network: one is to set up an independent recovery network in the area where products are concentrated as scrap and the other is to rationalize forward logistics nodes and make them function as recovery logistics nodes at the same time [11]. The operation mode of recovery outlets can be divided into independent operation of production enterprises, joint operation of production industries, and entrusted operation of third-party logistics enterprises according to different responsible subjects.

(2) Recycling centers: a recycling center as the intermediate link to undertake logistics tasks; its core function is to connect the recovery valve point and the processing end point, but it also has the functions of sports products storage, classification, and transportation. The operation efficiency of the recycling center plays a crucial role in the production plan of the processing end point [12]. For sports products collected and distributed from the recycling outlets, the recycling center will classify them according to different recycling and processing directions and then carry out storage and transportation operations in different ways until the sports products enter the processing terminal enterprises.

(3) Processing end point: the processing center is the final node in the recycling logistics system and the final target link of the recycling logistics system. According to the different processing methods of sports products, the processing end points can be divided into repair processing end points, remanufacturing processing end points, and comprehensive processing end points. However, its core and goal are to realize the recycling of resources through the harmless treatment of sports products.

3.2. Network Structure Analysis. The structure of the recovery logistics network can be expressed as a four-layer network structure of the collection and distribution layer, the recovery layer, and the processing and recycling layer according to the different logistics direction and node operation mode. The collection and distribution layer and the recovery layer, as the core, and layers of the recovery logistics system undertake the function of building the recovery logistics network [13]. The sports product recovery logistics network can be expressed as an upper, middle, and lower three-layer structure according to the order of logistics links. The lower layer is the recovery network to meet the needs of sports product distribution. The middle level is a recycling center, which carries out logistics operations of sports products. The upper layer is the sports product processing enterprises, which can realize the function of resource recycling. Therefore, the recycling logistics network can be expressed as the structure shown in Figure 2.

The underlying network structure of sports product recycling logistics is recycling $N$ points, whose main function is to carry out the preliminary collection of sports products. Therefore, according to the different sports products 


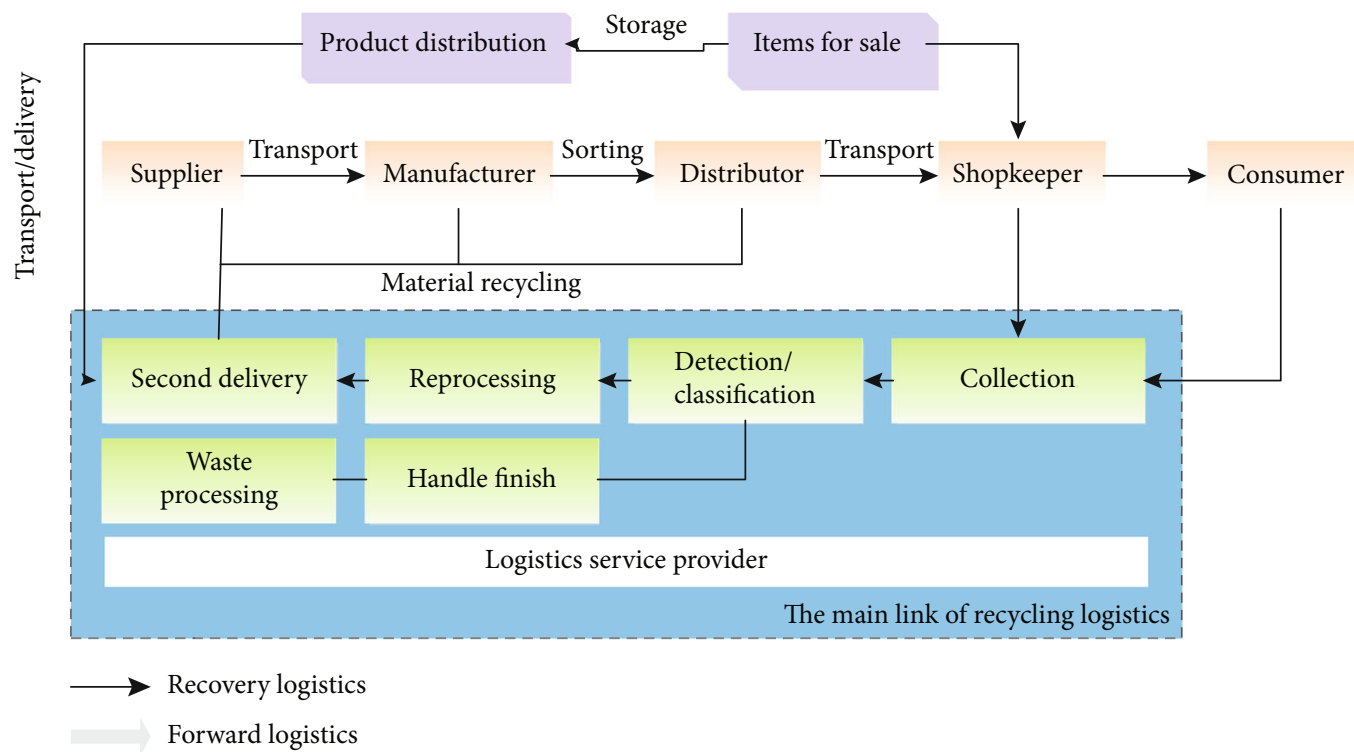

FIGURE 1: Recycling logistics business flow chart.

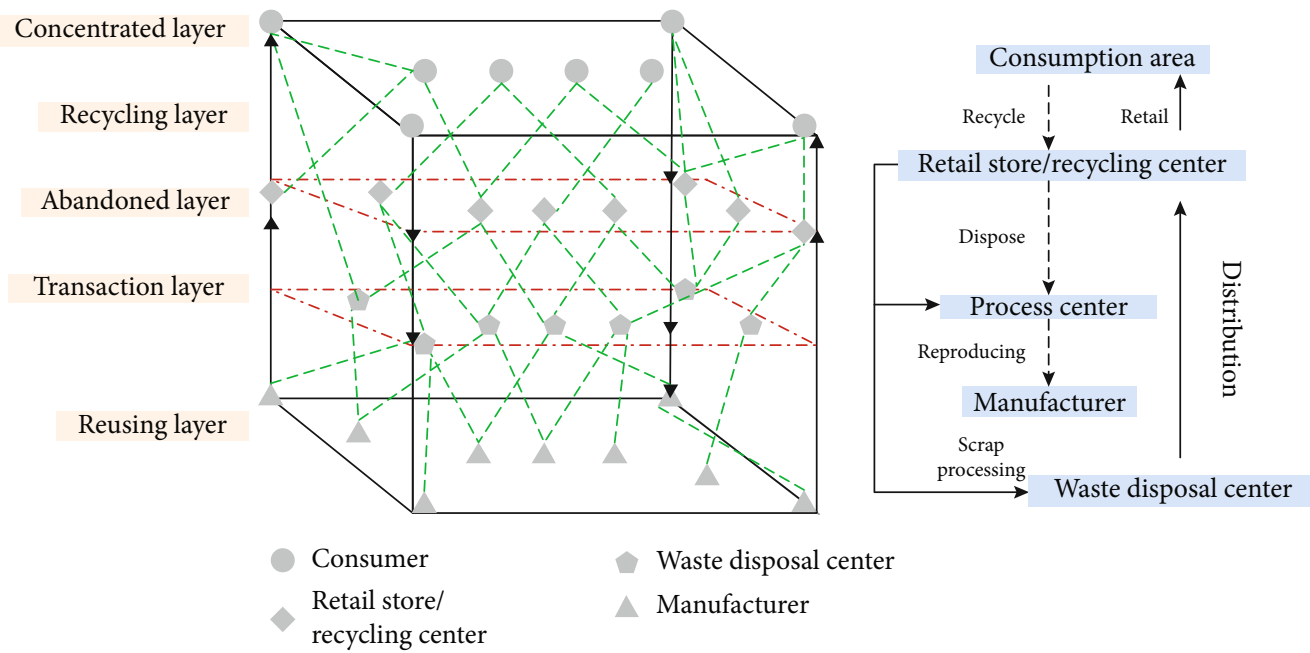

FIGURE 2: Sports good recycling logistics network structure.

recovered, generally, the recycling points are arranged to produce sports products with large frequency and large quantity. After completing the primary collection and distribution task of sports products in the recovery network, the sports products will continue to be concentrated again to the upper layer of the network structure, namely, the recovery center, through vehicles. The recycling center is an important link and node in the recycling network. By means of logistics operations such as recycling center, storage, classification, special product packaging, and transportation, it continues to transport to the processing center in the recycling network of sports products and finally reaches the processing node of sports products at the top of the network. For the sports product return flow network, after a reasonable layout of network recycling, recycling, and processing, the network nodes in the transportation operation link are also an important link in determining the operating efficiency and logistics cost of the recycling network, especially the focus of the recycling network to the recycling center. Whether it is for reasonable configuration and route planning, or to reduce transportation costs or recover the maximum profit, the recycling network structure is determined under the conditions of the main research content.

3.3. Management of Recycling Logistics by the Internet of Things. Perception technology [14], data processing technology, satellite positioning technology [15], and visual display technology [16] are used to manage and control the operation of the business process of recovery logistics, making the operation of the recovery logistics system more efficient. The technology used in this system is shown in Figure 3.

The Internet of Things technology has outstanding performance and powerful application ability in the field of recycling logistics. Because the Internet of Things application has to be able to have good perception, noncontact processing, good interaction with information, achieve excellent 


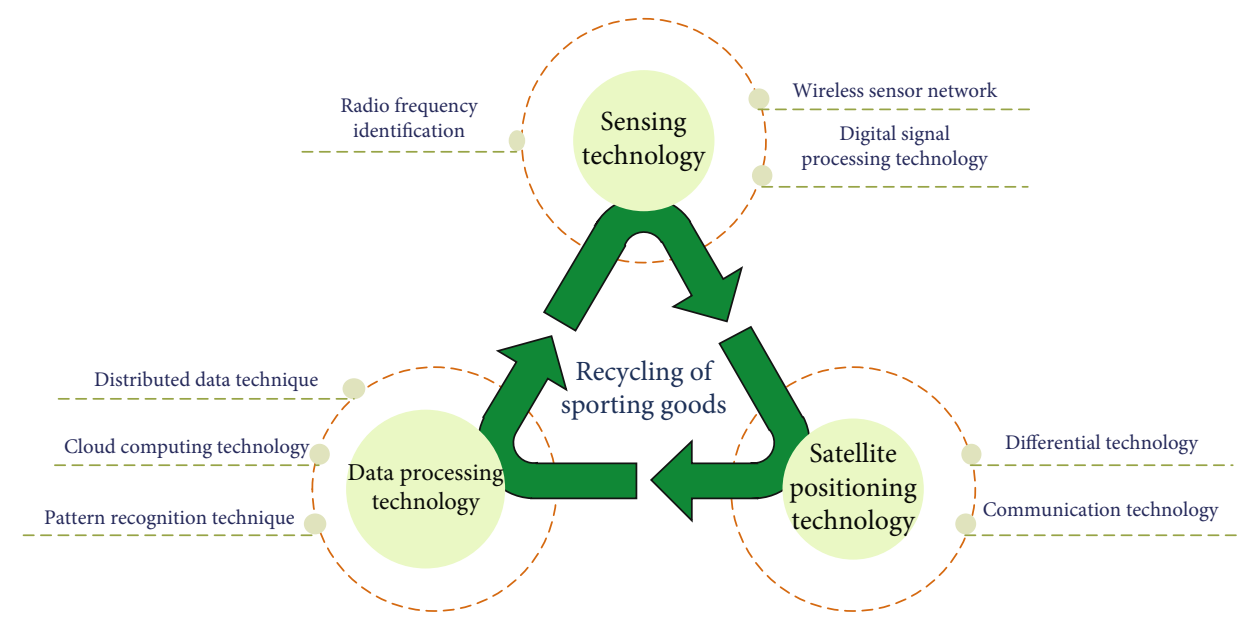

FIGURE 3: IoT technology applied in recycling systems.

feedback and control of technical characteristics and development of the logistics link mainly around items and objects, and rapid response and feedback of the item, the Internet of Things technology can effectively crack information barriers in the process of logistics operation, accelerate the speed of the logistics system, logistically link operations to reduce costs, and improve logistics efficiency of overall system performance, which embody in the following aspects: (1) improve the recycling logistics business link effective monitoring, (2) improve the efficiency of logistics operation, and (3) realize digital management.

\section{Sporting Good Recycling Management Platform}

4.1. Target Positioning and Demand Analysis. Based on recycling logistics information demand, combined with the Internet of Things for the positive influence and significance of returned logistics management, in view of the returned logistics system of main business, with the aid of returned logistics building method based on Internet of Things, around the recovery and recycling of sports products returned logistics information platform construction, the main content of the information platform for the design of the overall Japanese standard is summarized as the following aspects: (1) for the returned logistics management information platform to build good foundation for the environment; (2) build a recycling information docking platform to realize the wholeprocess control of recycling business; (3) integrate information resources of all links of recycling logistics to realize information resource sharing among transportation enterprises, recycling enterprises and sports product processing enterprises; (4) provide information support for logistics recovery and resource recycling operation control; and (5) achieving sustainable utilization of renewable resources and developing a circular economy.

According to the management departments and enterprises involved in the recovery logistics system, the information construction of the recovery logistics system is carried out according to the business and technical requirements, so as to realize the comprehensive management and operation control of the recovery logistics. In addition, the construction of the information platform also involves some nonfunctional requirements of the platform [17]. Therefore, the platform demand analysis can be divided into four aspects: user demand analysis, functional demand analysis, technical demand analysis, and nonfunctional demand analysis, as shown in Table 1.

For the management departments and related enterprises involved in the process of recycling logistics, based on the theory of the Internet of Things, the application of relevant information technology is carried out to construct the information of recycling logistics. Technical requirements can be divided into system integration technology, SOA architecture technology [18], cloud computing technology, data warehouse and data mining technology, and system interface technology.

(1) Information collection and transmission technology: perception and identification technology. This technology includes GPS, GIS, and bar code technology [19-23], which is used to realize the positioning, tracking, and monitoring of participating transportation vehicles and recovered goods. Network and communication technology is used to realize the interconnection, high-speed transmission, and sharing of information among monitoring units, enterprises, and individuals in the recovery logistics network, which provides technical support for the intelligent implementation of recovery operation planning and scheduling management of logistics vehicles.

(2) Transportation operation optimization technology: logistics optimization technology is an important technology to realize sports product recovery business. This technology mainly includes vehicle scheduling technology, vehicle path optimization technology, and cooperative processing technology of forward logistics operation task.

(3) Data analysis and mining technology is used to establish the virtual data warehouse which is used to solve 
TABLE 1: Recycling management system information construction requirements.

\begin{tabular}{lcccc}
\hline System needs & & & & \\
\hline User needs & Management department & Related enterprises & & Items \\
Functional needs & Network management & Warehouse management & Sorting management & $\begin{array}{c}\text { Transportation } \\
\text { management }\end{array}$ \\
Technical needs & $\begin{array}{c}\text { System integration } \\
\text { technology }\end{array}$ & SOA architecture technology & Cloud computing technology & Data mining technology \\
$\begin{array}{l}\text { Nonfunctional } \\
\text { needs }\end{array}$ & $\begin{array}{c}\text { Platform interface } \\
\text { requirements }\end{array}$ & $\begin{array}{c}\text { Hardware and software } \\
\text { requirements }\end{array}$ & $\begin{array}{c}\text { Environmental protection } \\
\text { requirements }\end{array}$ & $\begin{array}{c}\text { Economic and technical } \\
\text { index }\end{array}$ \\
\hline
\end{tabular}

the problem of how to read information from the heterogeneous data source skill which is a structured data environment, data warehouse, and data transmission via the Internet data analysis of rationalization, which is the application of different themes and decision-making support to achieve the ultimate goal. A data warehouse can well integrate different data sources and can update the subject data in real time, which can achieve the corresponding goals stably. Platform architecture technology includes system comprehensive integration technology, system interface technology, and SOA architecture technology $[24,25]$.

4.2. System Framework. Analyzing the return to logistics network characteristics of the Internet of Things, we must consider platform information requirements, logistics product requirements, and commercial transportation, as shown in Figure 4.

(1) Core business layer: core business is the core of the platform to implement recovery logistics business management, including recovery network management, transportation management, storage management, and classification management. Recycling network management refers to the unified management of each recycling network connected to the platform to collect and process the recycling information. The transportation management mainly manages the daily recycling transportation plan and the transportation operation process; warehouse management is used to meet the storage needs of the recycled sports products due to temporary storage. Classified management is mainly aimed at determining the flow direction of the recycled sports products according to the categories, so as to facilitate them to better enter the recycling system and transport to the corresponding recycling enterprises.

(2) Auxiliary business layer: auxiliary business is the core of the platform and the supporting business of business development, mainly including customer management, vehicle management, and equipment management. Customer management refers to the management of centralized waste production units, recycling enterprises, logistics enterprises, and other platform customers that participate in recycling activities. Through customer evaluation and statistical analysis, it provides high-quality customer recommendation and other services for upstream and downstream customers. Vehicle management is to manage the information of the quantity, type, load, in-transit state, and driving state of the transport vehicle. Equipment management mainly realizes the management of logistics equipment.

(3) Value-added business layer: value-added business layer includes four parts: e-commerce, logistics information service, recovery fee settlement, and sports product information service. Through the development of e-commerce, the platform provides product information services for recycling enterprises and centralized waste production units and provides a platform for trading information sharing and collection. Logistics information service refers to the platform that forms a recovery or transportation plan by integrating e-commerce order information, provides logistics enterprises with transportation information of recovered goods, and optimizes and improves the utilization rate of logistics enterprises' resources

4.3. Platform Construction. In the development of recycling logistics information platform for sports lead storage sports goods, the development environment and operation environment are shown in Table 2.

According to the recycling business process of sporting goods, combined with the content of the information platform framework, the recycling platform of sporting goods is developed. With recycling network as the core, the sports good recycling platform emphasizes the comprehensive control of the whole process of recycling, storage, transportation, and production of sports goods. The specific functions include the following: various ways to display and query the recovery dot; use of IoT related technology to manage the outbound and inbound operations of goods and inventory checking process; based on the intelligent management directly oriented to the specific logistics transportation and distribution command and operation level, the vehicle scheduling and operation plan are completed by using the scheduling optimization model, and a variety of advanced technologies are used to intelligentize the recovery and transportation process. In view of the related customers involved in the recovery, the establishment of customer management 


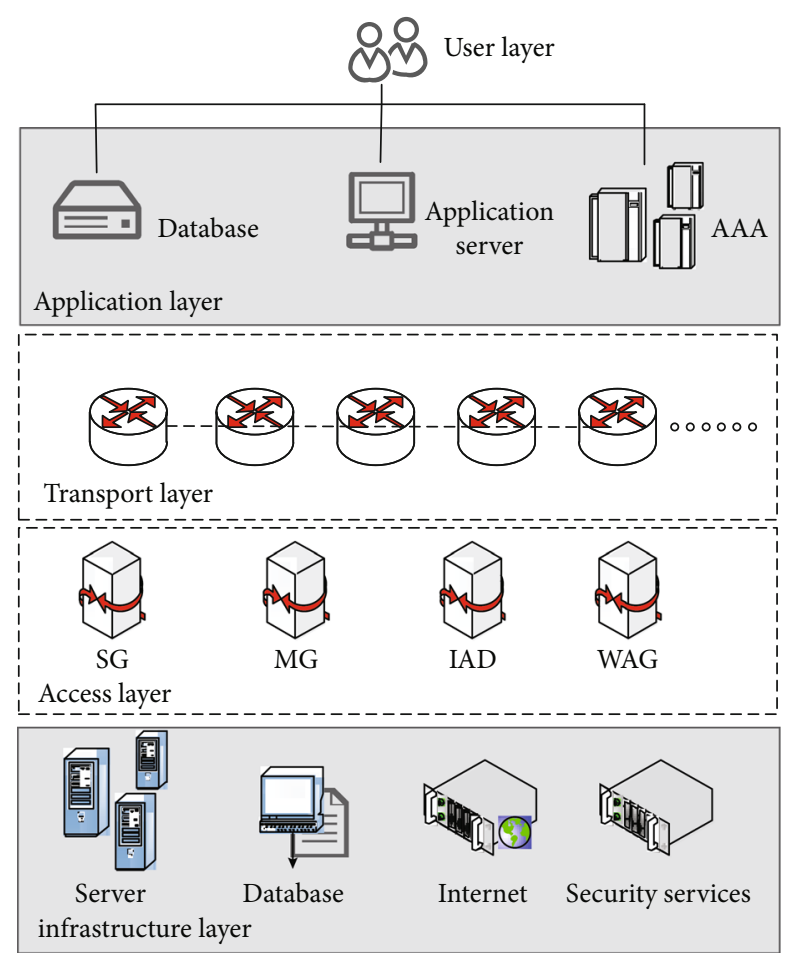

FIGURE 4: Sporting good recycling system framework.

system, to achieve the classification of customer management and improve the efficiency of customer management, use human resource management system to improve the efficiency of human resource management. Through the information platform, sports good information will be displayed to customers, so that merchants and customers can negotiate, trade, and pay online, and at the same time, the transaction process and payment process of both sides can be managed. At the same time, the emergency support system can be used to give early warning to possible safety problems in the platform and make intelligent judgment and generate an emergency plan.

\section{Platform Optimization}

In this paper, reducing system energy consumption is regarded as an optimization objective, and the alternating direction multiplier algorithm is applied to solve the problem more efficiently. Using the $\mathrm{KNN}$ algorithm to classify sports goods, the cloud model theory is introduced to identify unstable data partitions in unstructured data management systems.

\subsection{Optimization of Sports Good Classification Module. The} garbage classification module is responsible for inputting the images processed by the image preprocessing module into the machine learning model and outputting the final classification results. In this scheme, the machine learning model based on Lenet-5 is finally adopted. A conventional Lenet- 5 has seven layers, each containing multiple parameters, totaling more than 60,000 parameters. The number of parameters of the model increases proportionally with the size and number of convolution cores and exponentially with the number of layers of the model. Therefore, the scheme is simplified to remove one convolution layer and the pooling layer. The overall process is shown in Figure 5. The image extraction process is as follows. First, features are extracted through the convolution unit (each convolution unit includes a convolution layer and a sampling layer), and finally, all local features are summarized by the full connection layer.

The neuron multiplies the input signal with the corresponding weight value, adds the bias term, and finally outputs the final result through the activation function, which can be expressed by the following formula:

$$
y_{j}=f\left[\lim _{n \rightarrow \infty} \sum_{i=1}^{n}\left(x_{i}+b_{j}\right)\right],
$$

wherein $y_{j}$ is the activation function, which is used to determine whether the neuron should be activated under the current input, that is, whether the previous input can affect the next layer. In the training process, due to the inaccurate parameters of the model, the value of the output layer is usually different from the expected value. In this case, we need the back propagation (BP) algorithm to update the parameters of the model.

$$
E=e^{-\lim _{n \rightarrow \infty} \sum_{i=1}^{n}\left(y_{i}-\bar{y}_{i}\right)} .
$$

$E$ represents the sum of the square deviations of all $N$ sample errors, which is usually used as the objective function. Thus, it can be seen that the training of the model is to find the appropriate weight and bias the term to make the objective function take the minimum value, which is essentially a mathematical optimization problem. A gradient descent algorithm is used to calculate the partial derivative of the loss function. The gradient descent algorithm is used to calculate the partial derivative of the loss function. Take the $y$-th neuron in the NTH layer as an example again, and the $i$-th weight update formula of the upper layer is

$$
\omega_{i j}^{l-1}=\eta \cdot \frac{\partial E}{\partial \omega_{i j}^{l-1}}+y_{j}
$$

where $\eta$ is the learning rate that can be set.

After many experiments, the number and size of the convolution kernel are customized, so that the total number of parameters in this model is reduced to more than 5,000 . We set the size of the model as an upper limit of $60 \mathrm{kB}$, tested the classification accuracy of the model for four kinds of garbage under different parameters, and obtained the results as shown in Figure 6.

After the preprocessing module, the size of the image is $60 * 60$. However, if this size is used as the standard for subsequent processing of the model, the terminal will not be able to fully store the parameters required by the model. Therefore, we halved its resolution, that is, we took the average value of each $2 * 2$ region of the image, converted it into a $30 * 30$ image, and then filled a layer of 0 in its outer ring to obtain a $32 * 32$ image, which is convenient for subsequent processing operations. $5 * 11 * 11$ convolution kernels are 
TABLE 2: Information platform development environment and operation environment.

\begin{tabular}{lccc}
\hline System development environment & & System operating environment & \\
\hline Operating system & Windows 10 & Operating system & Windows XP \\
Development tools & MyEclipse & Browser & IE 10, etc. \\
Server & Apache 2.4.10 & Configuration & phpStudy 2010 \\
Database & MySQL 5.5.40 & & \\
Development platform & phpDesigner 7 & & \\
\hline
\end{tabular}

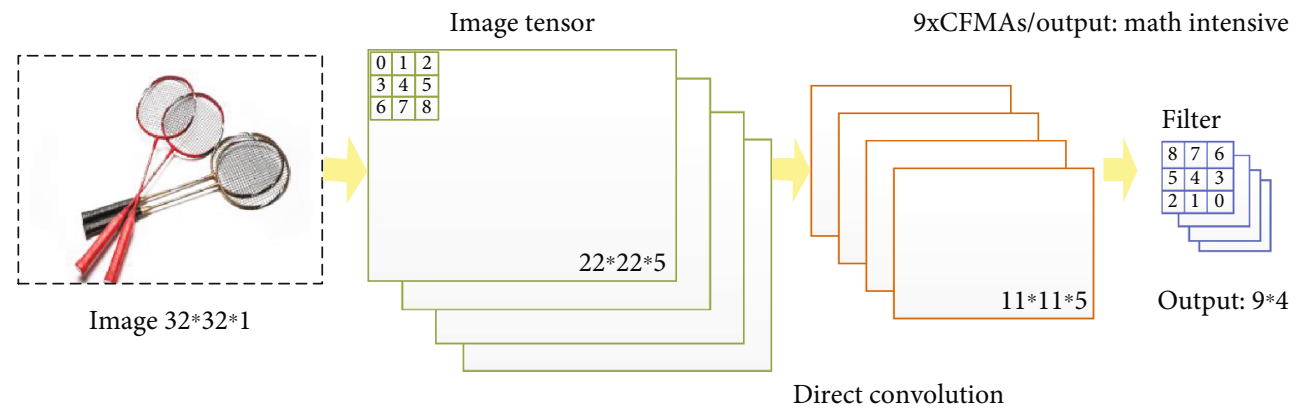

FIGURE 5: Sporting good classification model.

used to convolve the image to obtain a $22 * 22 * 5$ feature map. In this case, the number of parameters to be trained is $5 *(11 * 11+1)=610$, including each offset term. The original Lenet-5 adopts a Euclidean radial basis function, and each kernel represents a classification result. The category of the value with the smallest output value is the final classification result. In this scheme, considering the specific transplantation, it is changed into the ReLU activation function, and the largest value among the 4 output values is the classification result, with a total of $(8+1) * 4=36$ parameters.

5.2. Timely Delay Optimization of System Stability. The advantage of the system architecture lies in the rational use of the characteristics of the edge server and cloud server, that is, the edge server has general storage capacity and computing capacity but short network delay and stable communication. The cloud server has strong storage capacity and computing capacity, but the network delay is long and the communication is unstable. For the unstructured data management system, the system carries out cloud modeling for the data partitions in the storage system, analyzes the correlation between the data partitions, identifies the unstable data partitions, and rearranges them, so as to reduce the data transmission cost caused by frequent data movement in the unstructured data management system.

The cloud model theory is introduced to identify unstable data partitions in unstructured data management systems. The transmission time of the passive pull-out data set corresponding to the data partition is used as the criterion to identify the unstable data partition. The total transfer time overhead of the data set $d_{k}$ stored in the data partition $d p_{j}$ on node $S_{i}$ is

$$
\cos t\left(s_{i}, d p_{j}, d_{k}\right)=\lim _{n \rightarrow \infty}\left(\frac{\sum_{i=1}^{n}\left(\operatorname{size}_{k} / b_{j}\right) \cdot r_{i}^{i+k}-d_{k}}{\sum_{i=1}^{n}\left(\operatorname{size}_{k} / b_{j}\right) \cdot r_{i}^{i+k}+d_{k}}\right) .
$$

A cloud model for each data partition is needed first. The cloud model for constructing data partition is as follows:

$$
\begin{gathered}
E_{x}=\lim _{n \rightarrow \infty} \frac{1}{N+1} \sum_{I=1}^{N} u^{2}-1, \\
E_{n}=\lim _{n \rightarrow \infty} \sqrt{\mid \frac{1}{N+1}\left(\sum_{I=1}^{N}\left(u+E_{x}\right)^{2}-\sum_{I=1}^{N}\left(u-E_{x}\right)^{2} \mid-\sqrt{\frac{E_{x}}{2}}\right.},
\end{gathered}
$$

where $N$ is the number of cloud drops in a cloud model and $u$ is each cloud drop in the cloud model. After the cloud model of each data partition is obtained, the data partition is divided into the Stable Data Partition Group (SG) and the Unstable Data Partition Group (UG) based on the cloud model of each data partition. The ECM calculation formula of cloud model similarity is

$$
\operatorname{ECM}(\mathrm{DPC} 1, \mathrm{DPC} 2)=\frac{E_{x}}{\sqrt{2 / \pi}\left(E_{n}+E_{x}\right)^{2}} .
$$

To demonstrate the characteristics of the different servers, this article compares edge servers with cloud servers. The communication delay and communication stability of the two kinds of servers are, respectively, compared from the horizontal and vertical aspects. As can be seen from Figure 7 , the communication stability of the edge server is basically 2.5 times that of the cloud server, and the communication delay of the cloud server is 7 times that of the edge server, no matter in which day or in which time of day. The edge server is only one hop away from the device, while the cloud server is many hops away. Obviously, the edge server is more suitable for direct communication with the intelligent terminal and is responsible for some services requiring 


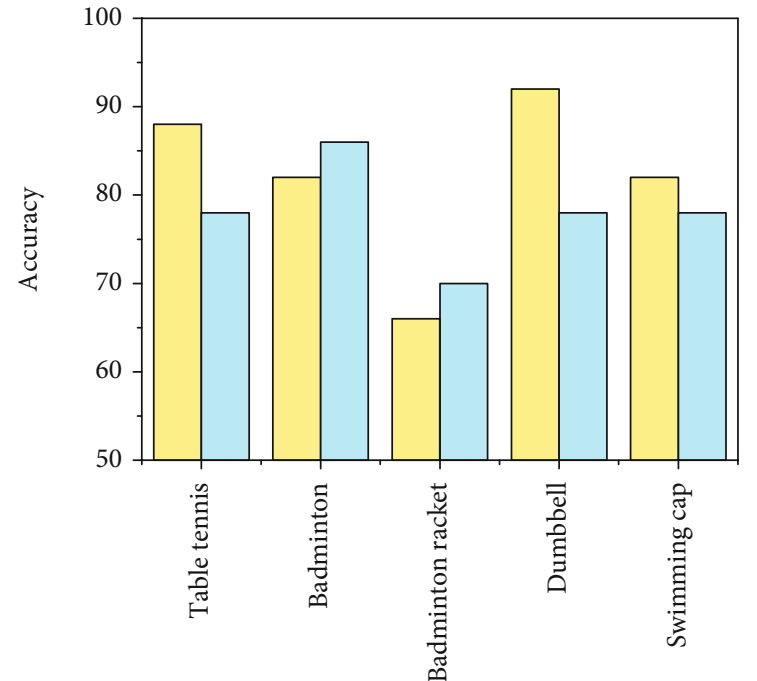

C1: $11 * 11 * 4$ C3: $11 * 11 * 12$

$\mathrm{C} 1: 9 * 9 * 5 \mathrm{C} 3: 12 * 12 * 16$

Figure 6: Model classification accuracy under different convolution kernels.

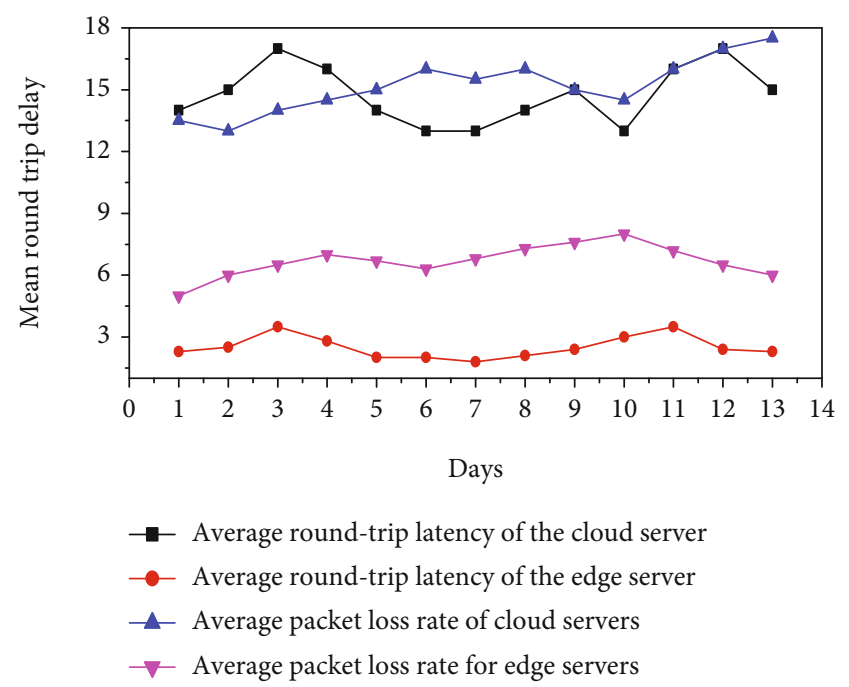

Figure 7: Comparison diagram of the performance of the two server networks.

stability and timeliness, while the cloud server is suitable for some services requiring a lot of computation and insensitive time delay.

5.3. Optimization of Path Planning Algorithm. The path planning algorithm based on genetic algorithm designed in this system comprehensively considers three factors, namely, the degree of recycling bucket filling, the full time, and the time to wait for recycling. We compare it with the distancefirst path planning algorithm. The distance-first algorithm only considers the distance between each recycling bucket and sorts the distance between each recycling bucket in order from small to large. According to this order, the final recy-

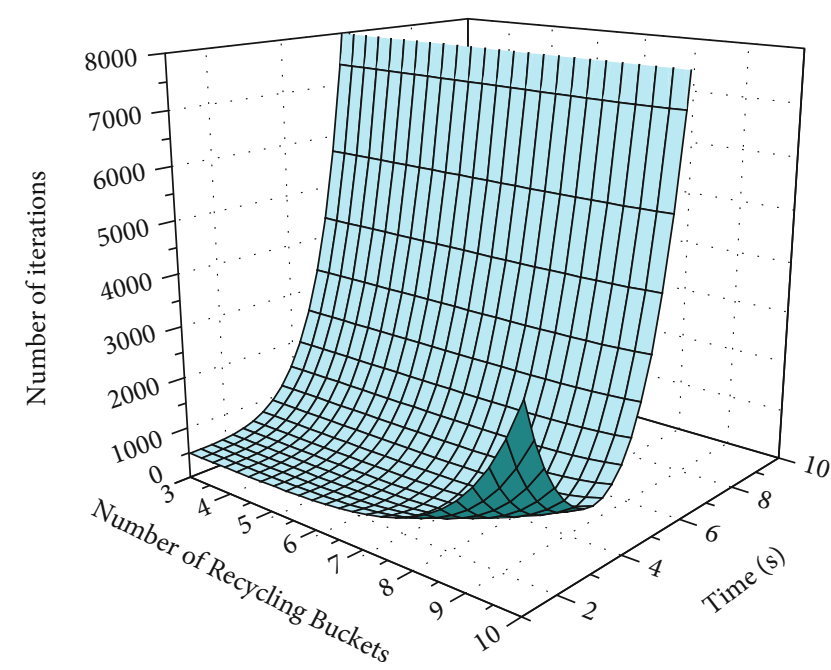

FIGURE 8: The relationship between the number of iterations, time, and number of recycling barrels.

cling and cleaning path can be obtained. After the path planning algorithm based on the genetic algorithm obtains the final path, the path obtained by the distance-first algorithm is substituted into the fitness function to obtain the fitness values of the two paths, respectively. The path with a smaller fitness value is the better path. The comparison shows that the improved genetic algorithm is superior to the distance first algorithm in any case.

This system also evaluates the time complexity of the path planning algorithm based on the genetic algorithm, and the influence of different parameters on the algorithm, Figure 8, is the number that shows the recycling barrel and the influence of the number of times of iteration algorithm running time; we can find 205000 trash iteration times, with the algorithm of the running time of 5 seconds; it has to do with paltry garbage pickup compared to the time saved. Figure 8 shows the convergence ability of the algorithm under different iteration times. We can find that the fitness value will not change after 5000 times, that is, the algorithm gets the optimal solution, so the number of iterations of the algorithm is also set to 5000 .

\section{Conclusion}

Based on the analysis of the current situation and existing problems of the returned good flow, combined with the characteristics of the returned good flow process and the recycling network structure, this paper proposes to use the powerful intelligence of the Internet of Things and efficient information transmission capabilities and good operation and maintenance capabilities. We combine the return flow business and links, design an information framework platform, and develop and design an information platform framework based on the actual physical recycling business background. Combined with the feedback of the actual operation of the platform, the functions that can be realized in the optimization of the recovery business path are proposed. By establishing the corresponding model, the overall framework of the information platform is proposed from a macro perspective, 
with business activities as the main management object and with the help of target positioning. Through the inspection of its operation process, network structure, and management objects, on the basis of the framework, computer technology is used for development and implementation. Through the design, development, and optimization of some functions of the information platform, the positive role of Internet of Things technology in improving the efficiency and reducing the cost of recovery logistics business management has been confirmed, and the ideas for solving the existing problems in recovery logistics management have been provided.

\section{Data Availability}

The data used to support the findings of this study are available from the corresponding author upon request.

\section{Conflicts of Interest}

The authors declare that they have no known competing financial interests or personal relationships that could have appeared to influence the work reported in this paper.

\section{Acknowledgments}

The authors received the following funding: Henan Provincial Soft Science Research Plan Project: Research on the Governance Path of Social Sports Organizations Participating in Rural Public Sports Service Supply under the Concept of Shared Development (Project No.: 202400410261); the General Project of the "13th Five-Year Plan" of Education Science in Henan Province: Research on the Path of Tailor Poverty Reduction of School Physical Education in Deep PovertyStricken Areas under the Concept of Shared Development (Project No.: 2019-JKGHYB-0331).

\section{References}

[1] S. Pimenta and S. T. Pinho, "Recycling carbon fibre reinforced polymers for structural applications: technology review and market outlook," Waste Management, vol. 31, no. 2, pp. 378392, 2011.

[2] J. Rifkin, The Zero Marginal Cost Society: The Internet of Things, the Collaborative Commons, and the Eclipse of Capitalism, St. Martin's Press, 2014.

[3] E. Manavalan and K. Jayakrishna, "A review of internet of things (IoT) embedded sustainable supply chain for industry 4.0 requirements," Computers \& Industrial Engineering, vol. 127, pp. 925-953, 2019.

[4] F. K. Shaikh, S. Zeadally, and E. Exposito, "Enabling technologies for green internet of things," IEEE Systems Journal, vol. 11, no. 2, pp. 983-994, 2015.

[5] W. Hou, W. Li, L. Guo, Y. Sun, and X. Cai, "Recycling edge devices in sustainable internet of things networks," IEEE Internet of Things Journal, vol. 4, no. 5, pp. 1696-1706, 2017.

[6] I. Mahmood and J. A. Zubairi, "Efficient waste transportation and recycling: enabling technologies for smart cities using the internet of things," IEEE Electrification Magazine, vol. 7, no. 3, pp. 33-43, 2019.
[7] W. Ren, Y. Sun, D. Zhao et al., "High-performance wearable thermoelectric generator with self-healing, recycling, and Lego-like reconfiguring capabilities," Science Advances, vol. 7, no. 7, article eabe0586, 2021.

[8] H. N. Saha, S. Auddy, S. Pal et al., "Waste management using internet of things (iot)," in 2017 8th annual industrial automation and electromechanical engineering conference (IEMECON), pp. 359-363, Bangkok, Thailand, 2017.

[9] A. Angani, J. C. Lee, and K. J. Shin, "Vertical recycling aquatic system for internet-of-things-based smart fish farm," Sensors and Materials, vol. 31, no. 12, pp. 3987-3998, 2019.

[10] Z. Zhou, Y. Cai, Y. Xiao, X. Chen, and H. Zeng, "The optimization of reverse logistics cost based on value flow analysis - a case study on automobile recycling company in China," Journal of Intelligent \& Fuzzy Systems, vol. 34, no. 2, pp. 807-818, 2018.

[11] E. Ingemarsdotter, E. Jamsin, G. Kortuem, and R. Balkenende, "Circular strategies enabled by the internet of things-a framework and analysis of current practice," Sustainability, vol. 11, no. 20, p. 5689, 2019.

[12] R. Ramly, A. A. Bakar Sajak, and M. Rashid, "IoT recycle management system to support green city initiatives," Indonesian Journal of Electrical Engineering and Computer Science, vol. 15, no. 2, pp. 1037-1045, 2019.

[13] O. Elsayed, M. Abouzied, V. Vaidya, K. Ravichandran, and E. Sanchez-Sinencio, "An ultralow-power RF wireless receiver with RF blocker energy recycling for IoT applications," IEEE Transactions on Microwave Theory and Techniques, vol. 66, no. 11, pp. 4927-4942, 2018.

[14] F. Benkhelifa, H. ElSawy, J. A. Mccann, and M. S. Alouini, "Recycling cellular energy for self-sustainable IoT networks: a spatiotemporal study," IEEE Transactions on Wireless Communications, vol. 19, no. 4, pp. 2699-2712, 2020.

[15] H. Wang, H. Han, T. Liu et al., "'Internet +" recyclable resources: a new recycling mode in China," Resources, Conservation and Recycling, vol. 134, pp. 44-47, 2018.

[16] T. J. Sheng, M. S. Islam, N. Misran et al., "An internet of things based smart waste management system using LoRa and tensorflow deep learning model," IEEE Access, vol. 8, pp. 148793-148811, 2020.

[17] F. Gu, B. Ma, J. Guo, P. A. Summers, and P. Hall, "Internet of things and big data as potential solutions to the problems in waste electrical and electronic equipment management: an exploratory study," Waste Management, vol. 68, pp. 434-448, 2017.

[18] G. C. Nobre and E. Tavares, "Scientific literature analysis on big data and internet of things applications on circular economy: a bibliometric study," Scientometrics, vol. 111, no. 1, pp. 463-492, 2017.

[19] M. Maksimovic, "Leveraging internet of things to revolutionize waste management," International Journal of Agricultural and Environmental Information Systems, vol. 9, no. 4, pp. 113, 2018.

[20] N. Salah and M. Rabee, "Smart recycle bin system based on Wi-Fi and IoT," International Journal of Computer Applications, vol. 181, no. 4, pp. 34-37, 2018.

[21] S. Keivanpour and D. A. Kadi, "Internet of things enabled realtime sustainable end-of-life product recovery," IFAC-PapersOnLine, vol. 52, no. 13, pp. 796-801, 2019.

[22] J. Han, N. Lin, J. Ruan, X. Wang, W. Wei, and H. Lu, “A model for joint planning of production and distribution of fresh produce in agricultural internet of things," IEEE Internet of Things Journal, 2020. 
[23] W. Wang, N. Kumar, J. Chen et al., "Realizing the potential of the internet of things for smart tourism with $5 \mathrm{G}$ and AI," IEEE Network, vol. 34, no. 6, pp. 295-301, 2020.

[24] W. Wei, M. Guizani, S. H. Ahmed, and C. Zhu, "Guest editorial: special section on integration of big data and artificial intelligence for internet of things," IEEE Transactions on Industrial Informatics, vol. 16, no. 4, pp. 2562-2565, 2020.

[25] S. H. Ahmed, V. H. C. de Albuquerque, and W. Wei, "Guest editorial: special section on advanced deep learning algorithms for industrial internet of things," IEEE Transactions on Industrial Informatics, vol. 17, no. 4, pp. 2764-2766, 2021. 